\title{
D-05
}

\section{EVALUACIÓN DEL COSTE DE ELIMINAR LA SOBREEXPLOTACIÓN DEL ACUÍFERO "ALTO GUADALENTÍN" MEDIANTE UNA OFERTA PÚBLICA DE ADQUISICIÓN DE DERECHOS DE AGUA}

\author{
Martínez-Granados, D (1) (P), Calatrava, J (2)
}

\begin{abstract}
1 Investigador, E.T.S. de Ingeniería Agronómica, Universidad Politécnica de Cartagena, Paseo Alfonso XIII 48, 30203 Cartagena, david.martinez@upct.es

2 Profesor Titular de Universidad, E.T.S. de Ingeniería Agronómica, Universidad Politécnica de Cartagena, Paseo Alfonso XIII 48, 30203 Cartagena, j.calatrava@upct.es
\end{abstract}

\section{Resumen}

La sobreexplotación de acuíferos es uno de los principales problemas ambientales y de gestión del agua en el sureste de España. En algunas zonas, el reciente desarrollo de la desalinización supone una oportunidad para hacer frente a este problema a un menor coste social y económico. Este trabajo analiza el impacto económico de eliminar, mediante la adquisición pública de derechos de aguas subterráneas, la sobreexplotación en el acuífero Alto Guadalentín, uno de los casos más graves de degradación de recursos subterráneos de España. Dicho impacto se evalúa mediante un modelo de programación matemática que maximiza el margen neto derivado de utilizar las diferentes fuentes de suministro de agua disponibles en la zona para el regadío. Nuestros resultados muestran cómo la adquisición pública de derechos de aguas subterráneas tiene un impacto económico similar a la restricción de las extracciones del acuífero, si bien en el primer caso es la Administración la que soporta el coste de recuperación del acuífero, mientras que en el segundo éste coste recae sobre los agricultores, lo que convierte a esta última en una medida más impopular y con un mayor coste político. En ambos casos, la disponibilidad de recursos desalinizados reduce significativamente este impacto, ya que los agricultores pueden sustituir recursos subterráneos por desalinizados.

PALABRAS CLAVE: Aguas subterráneas, cuenca del Segura, economía del agua, demanda de agua, programación matemática, regadío.

\section{1- Introducción}

La sobreexplotación de acuíferos es uno de los principales problemas ambientales y de gestión del agua en el Mediterráneo. En el caso de la cuenca del Segura, una de las regiones agrícolas más productivas de España, la expansión de la horticultura intensiva durante las últimas décadas ha incrementado las presiones sobre los recursos subterráneos, una de las principales fuente de suministro de agua de la cuenca, haciendo que la sobreexplotación haya alcanzado proporciones alarmantes en muchos acuíferos, lo que pone en riesgo el cumplimiento con los objetivos medioambientales establecidos para 2027 en cumplimiento con la Directiva Marco del Agua (DMA). Uno de los casos más graves de sobreexplotación y degradación de los recursos subterráneos, no solo de España sino de toda Europa, es el acuífero Alto Guadalentín, situado en los municipios de Lorca y Puerto Lumbreras en el suroeste de la cuenca del Segura.

Aunque la DMA pone el acento en la tarificación como instrumento para la gestión de los recursos hídricos, en general, en situaciones de sobreexplotación de acuíferos, no suele 
recurrirse a establecer tarifas o tasas sobre el agua, sino a la ordenación de los recursos subterráneos mediante cuotas fijas de bombeo, restricciones o zonificaciones. Diversos trabajos han analizado otras opciones como la restricción de extracciones mediante planes de gestión que incluyen el establecimiento de cuotas y su intercambio en mercados o bancos de agua, los pagos por reducir el consumo, la compra de derechos o la cooperación entre los usuarios del recurso (Iglesias, 2002; Hellegers y van lerland, 2003; Albiac et al., 2006 y 2008; Carmona et al., 2011; Esteban y Albiac, 2011).

La compra de derechos de agua de riego ha sido uno de los instrumentos más aplicados en España, a través del Plan Especial del Alto Guadiana y los centros de intercambio de derechos de uso del agua de las cuencas del Júcar y Segura (Calatrava y Gómez-Ramos, 2009; Garrido et al., 2013). La reforma de 1999 de la Ley de Aguas (Ley 46/1999) contemplaba la posibilidad de que los organismos de cuenca creasen los denominados Centros de Intercambio de Derechos (CID). A través de estos, los organismos de cuenca podrían realizar ofertas públicas de adquisición de derechos (OPAD) a los titulares interesados en ceder temporal o permanentemente sus concesiones para proceder posteriormente a su cesión a otros titulares (Calatrava y Gómez-Ramos, 2009). En el espíritu inicial de la Ley 46/1999 estaba que los CID funcionasen como mecanismo para agregar y poner en contacto oferta y demanda de agua, a la manera de los Bancos de Agua que funcionan en los Estados Unidos (Garrido et al., 2013), no permitiéndose que pudiesen retener los derechos adquiridos y obligándoles a ofertar todos, limitándose por tanto su papel al de intermediarios.

Los primeros CID se crearon en las cuencas hidrográficas del Guadiana, Júcar y Segura en 2004. Pese a ello, no funcionaron hasta que, con el inicio de la sequía de 2005-2008, el RDL 9/2006 reforzó su eficacia para que pudiesen dar respuesta también a otras demandas, principalmente ambientales. En concreto, además de ceder recursos a otros usuarios (objetivo inicial), los CID podrían también dedicar derechos adquiridos para usos ambientales o cederlos a las Comunidades Autónomas. De hecho, todos los CID han funcionado solo como adquirientes de agua o de derechos que no han sido vendidos a otros usuarios (Garrido et al., 2013). Los recursos adquiridos se han cedido a otros usuarios en forma de nuevas concesiones (caso del Guadiana) o (casos del Segura y Júcar) se han utilizado para mantener caudales ecológicos en épocas de sequía (Rey et al., 2014). Por lo tanto, la principal utilidad de los CID ha sido la protección ambiental, de manera similar al caso de los bancos públicos del agua de California, Australia o Canadá (Garrido et al., 2013; Calatrava y Martínez-Granados, 2016).

En el caso de la cuenca del Segura, la CHS lanzó dos OPAD en 2007 y 2008 con el objetivo de crear una reserva estratégica para garantizar los caudales ambientales en los ríos Segura y Mundo, en la parte alta de la cuenca. En ambos casos, el presupuesto de la OPAD era de 700.000 euros y la compensación máxima a pagar por la cesión de derechos era 0,18 $€ / \mathrm{m}^{3}$. Todos los caudales adquiridos se destinaron a usos ambientales (Garrido et al., 2013). Al igual que en el caso del Júcar, el éxito de las OPAD del Segura fue moderado, ya que no se llegó a agotar el presupuesto inicialmente establecido por falta de suficientes oferentes (Garrido et al., 2013; Rey et al., 2014). En opinión de Calatrava y Martínez-Granados (2016), el precio no era lo suficientemente atractivo, ya que apenas supera el valor marginal del agua en la zona calculado por Calatrava y Martínez-Granados (2012).

La adquisición de derechos de agua tiene la ventaja de que permite reducir permanentemente las extracciones, además de ser menos conflictivo que establecer restricciones o cuotas de consumo u otras políticas de "mando y control". Sin embargo, tiene un elevado coste presupuestario para la Administración y, al reducir el uso del agua, puede tener un importante impacto sobre el empleo y la economía de las zonas rurales. Carmona et al. (2011) muestran como, para conseguir una recuperación parcial de los niveles de los acuíferos en la cuenca del Alto Guadiana, es necesario restringir la disponibilidad de agua a 
los agricultores y establecer un precio de compra de los derechos de agua elevado. Sin embargo, el cumplimiento de las restricciones de agua reducirá los ingresos agrícolas sino se adoptan medidas adicionales para compensar dichas pérdidas.

A diferencia de otras zonas en las que se ha utilizado este instrumento, como es el caso del Alto Guadiana, en el caso del Alto Guadalentín, el reciente desarrollo de recursos desalinizados supone una oportunidad para hacer frente a este problema a un menor coste social y económico. El uso de recursos desalinizados es una de las opciones barajadas por la Administración hidráulica para hacer frente a la escasez de agua en zonas del sureste de España (Downward y Taylor, 2007), siendo también una de las defendidas por Albiac et al. (2006 y 2008) por presentar menores costes privados y sociales que otras alternativas. Sin embargo, el uso de recursos desalinizados puede no suponer en la práctica una alternativa efectiva para reducir la sobreexplotación de los acuíferos, debido a que el coste de los recursos provenientes de la desalinización es muy superior al de los subterráneos. Incluso en zonas en las que la agricultura puede asumir el coste de la desalinización, existe un incentivo para optar por la extracción de aguas subterráneas a un menor coste.

En este sentido, el objetivo de este trabajo es evaluar el impacto económico de eliminar la sobreexplotación en el acuífero Alto Guadalentín mediante la compra pública de derechos de aguas subterráneas, teniendo en cuenta la futura disponibilidad de recursos procedentes de la desalinización de agua de mar. Se analiza también la opción de restringir las extracciones de recursos subterráneos a su fracción renovable mediante el establecimiento de cuotas, una opción que ha sido considerada por la Administración pero que por su complejidad institucional y por su elevado impacto económico ha sido aparcada en beneficio de la opción del aporte de nuevos recursos externos a la zona.

\section{2- Regadío y sobreexplotación en el acuífero Alto Guadalentín}

El acuífero Alto Guadalentín está situado en el Valle del Guadalentín que se ubica en el suroeste de la cuenca del Segura en torno al río Guadalentín, un río-rambla que es el afluente más grande de la margen derecha del río Segura (Figura 1). El Guadalentín constituye una de las regiones agrícolas más productivas del país. La importancia del sector agrario en la zona se debe en gran medida a sus características climáticas, entre el clima Mediterráneo y el semiárido, que la convierten en un marco idóneo para la producción agrícola temprana (Calatrava et al., 2011). Sin embargo, esta zona presenta unas precipitaciones escasas, con medias que no alcanzan los $350 \mathrm{~mm} / \mathrm{año}$, provocando un fuerte déficit hídrico y frecuentes periodos secos.

La escasez estructural de agua es especialmente grave en la zona. Aunque la cuenca del Segura recibe recursos externos a través del acueducto Tajo-Segura (ATS), la notable expansión de la superficie de regadío desde que el ATS comenzase a funcionar en 1980 ha ocasionado un déficit crónico de agua en la cuenca del Segura en general y en el Alto Guadalentín en particular.

De acuerdo con nuestras estimaciones a partir de información obtenida del Sistema de Información Geográfica de la Confederación Hidrográfica del Segura (https://www.chsegura.es), la superficie total de regadío sobre el acuífero Alto Guadalentín supone 36.026 hectáreas, de las cuales 21.107 pueden ser regadas. La demanda agraria de agua asciende a 116,42 hm³/año, mientras que la disponibilidad de agua teórica de las diferentes fuentes de suministro se limita a $94.26 \mathrm{hm}^{3} / a n ̃ o$ (Tabla 1). En la práctica, este desequilibrio entre recursos y demandas es mayor. 


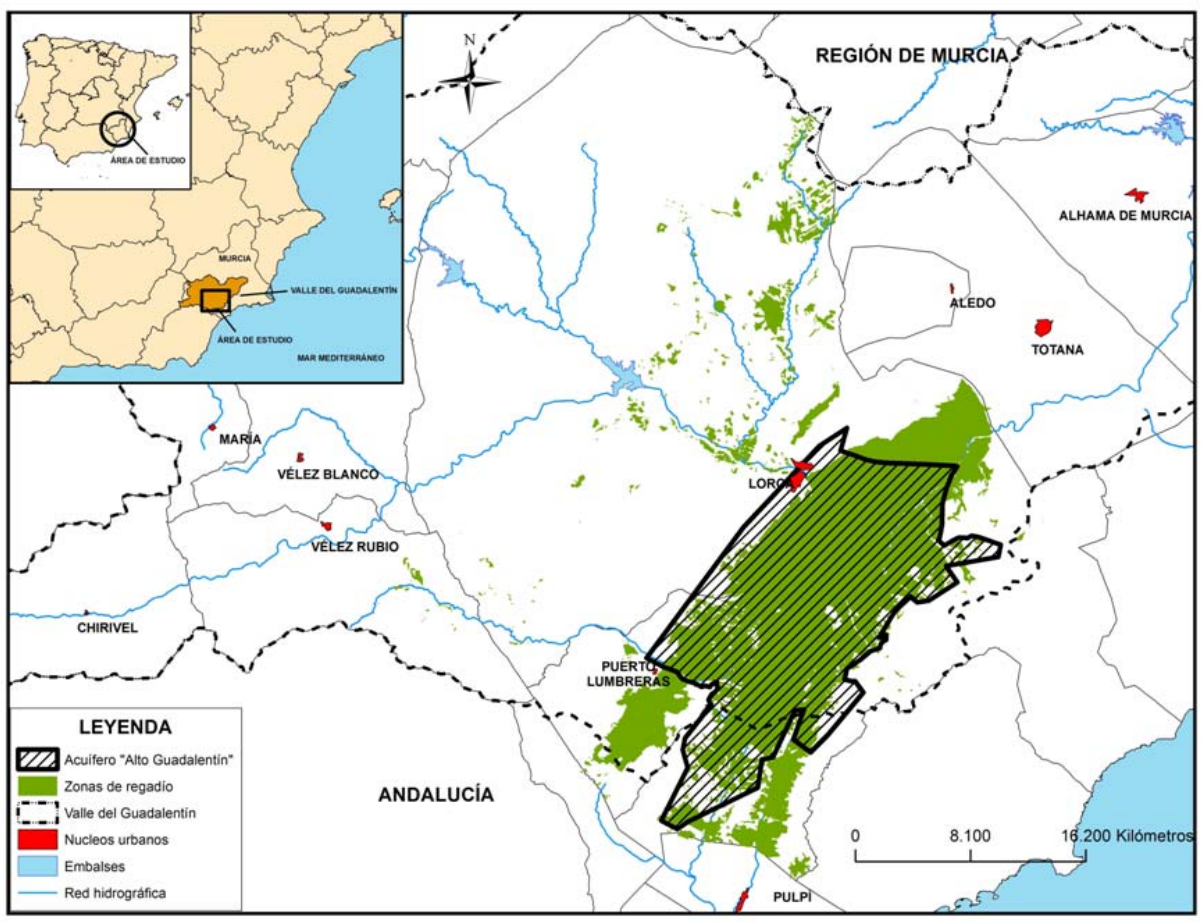

Figura 1. Ubicación y zonas regables del acuífero Alto Guadalentín.

Elaboración propia a partir del Sistema de Información Geográfica de la Confederación Hidrográfica del Segura (https://www.chsegura.es).

La disponibilidad teórica de agua se basa en los derechos y concesiones de agua existentes. En la práctica, la disponibilidad de recursos renovables se ha reducido desde los años ochenta del pasado siglo y el trasvase Tajo-Segura solo suministra una media del $60 \%$ de la cantidad anual máxima transferible para regadío. Por lo tanto, los recursos realmente disponibles se estiman en $74,35 \mathrm{hm}^{3} / a n ̃ o$, sin contar los recursos procedentes en un futuro próximo de la desalinizadora de Águilas (Tabla 1). Estos recursos actualmente utilizados cubren solo el 64\% de la demanda agraria (116,42 hm³/año), lo que supone un déficit de $42,1 \mathrm{hm}^{3} / a n ̃ o$ con respecto a las necesidades agronómicas de los cultivos. Este déficit se cubre en parte mediante la reducción de la superficie regada y en parte mediante la infradotación de los cultivos.

Tabla 1. Disponibilidad y coste de los recursos hídricos en el acuífero "Alto Guadalentín"

\begin{tabular}{|c|c|c|c|}
\hline Origen del recurso & $\begin{array}{l}\text { Derechos de agua } \\
\text { ( } \mathrm{hm}^{3} / \text { año) }\end{array}$ & $\begin{array}{l}\text { Recursos } \\
\text { disponibles } \\
\left(\mathrm{hm}^{3} / \text { año) }\right.\end{array}$ & $\begin{array}{c}\text { Coste del agua } \\
\left(€ / \mathrm{m}^{3}\right)\end{array}$ \\
\hline Superficiales & 13,90 & 11,10 & 0,050 \\
\hline Trasvase Tajo-Segura & 37,16 & 22,25 & 0.127 \\
\hline Reutilización & 4,70 & 4,70 & 0,080 \\
\hline Subterráneas & 38,50 & 36,30 & 0,240 \\
\hline Desalinización & - & 28,00 & 0,445 \\
\hline \multirow[t]{2}{*}{ Total } & $94,26^{*}$ & $74,35^{\star}$ & \\
\hline & renovables ( $\left.\mathrm{hm}^{3} / \mathrm{año}\right)$ & 27.60 & \\
\hline
\end{tabular}

Fuente: Elaboración propia a partir del Sistema de Información Geográfica de la Confederación Hidrográfica del Segura (https://www.chsegura.es) y de una encuesta realizada en 2012 a las comunidades de regantes de la zona. (*) No incluye los recursos procedentes de la desalación. 
En estas circunstancias, y dada la elevada rentabilidad del regadío en la zona, existe un incentivo muy grande para explotar los recursos subterráneos de la zona de manera insostenible, hasta tal punto que las aportaciones de aguas subterráneas suponen 36,3

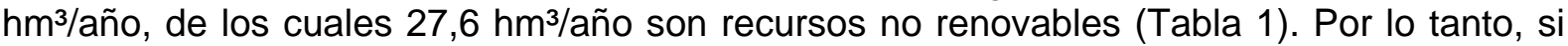

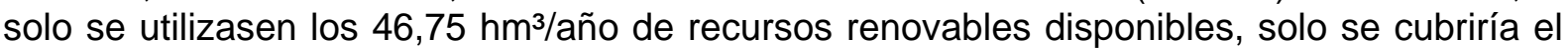
$40 \%$ de la demanda agraria de agua.

El uso masivo de recursos subterráneos durante las últimas décadas ha causado un serio problema de agotamiento del acuífero Alto Guadalentín, uno de los casos más graves de sobreexplotación de Europa. En base a la profundidad media del acuífero y a sus niveles piezométricos (CHS, 2008), estimamos que las extracciones no renovables acumuladas desde finales de los años setenta del pasado siglo suponen aproximadamente un $60 \%$ de la capacidad del acuífero. Además, el notable descenso de los niveles piezométricos ha incrementado los costes de bombeo en la zona y degradado la calidad del agua.

\section{3- Evaluación económica de los diferentes escenarios}

El impacto económico de los diferentes escenarios analizados se evalúa utilizando un modelo de programación matemática que maximiza el margen neto derivado de utilizar las diferentes fuentes de suministro de agua disponibles en la zona para el regadío y que permite simular el impacto económico de cada escenario.

La función objetivo del modelo es:

[1]

Sujeto a:

Donde o representa cada uno de los orígenes del agua disponible (superficial, trasvase Tajo-Segura, residual depurada, subterránea y desalinizada); wu es la cantidad de agua total utilizada en la zona contando todas las fuentes de suministro o ( $\left.\mathrm{hm}^{3} / \mathrm{año}\right) ; M N(w u)$ es una función que relaciona la cantidad de agua utilizada en la zona con el margen neto generado en la misma (euros); $p w_{o}$ es el coste medio de cada fuente de suministro $o$ (euros $\left./ \mathrm{m}^{3} / a n ̃ o\right) ; ~ w u_{o}$ es la cantidad de agua utilizada en la zona de cada fuente de suministro

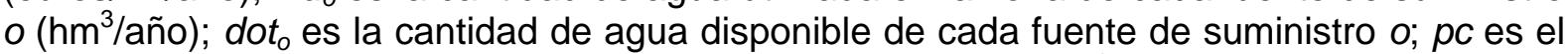
precio de compra de derechos de aguas subterráneas (euros $\left./ \mathrm{m}^{3} / a n ̃ o\right) ; ~ w v_{\text {subt }}$ es la cantidad de agua subterránea cuyos derechos son adquiridos por la administración ( $\left.\mathrm{hm}^{3} / a n ̃ o\right)$. Las variables de decisión del modelo son la cantidad de agua utilizada de cada fuente de suministro $w u_{o}$ (y por lo tanto $w u$ ) y la cantidad de derechos de aguas subterráneas adquiridas por la autoridad de cuenca $\left(w v_{\text {subt }}\right)$.

La función objetivo [1] maximiza el margen neto derivado de utilizar las diferentes fuentes de suministro de agua de regadío disponibles en la zona de estudio. La restricción [2] calcula la cantidad total de agua utilizada $\left(w_{u}\right)$ como la suma de las cantidades de agua utilizadas de 
cada origen $\left(w u_{0}\right)$. El conjunto de restricciones [3] restringe la cantidad de agua utilizada de cada origen $\left(w u_{0}\right)$ a su disponibilidad. La restricción [4] contabiliza las ventas de derechos de agua e impide que sean utilizados. Finalmente, las expresiones [5] y [6] son restricciones de no negatividad de las variables de decisión $\left(w u_{0}\right.$ y $\left.w v_{\text {subt }}\right)$.

El modelo se ejecuta para cada posible valor de $p c$ y se identifica el valor de dicho parámetro que permite eliminar los bombeos no renovables de agua. A partir de los valores óptimos de las variables de decisión $\left(w u_{0}{ }^{*}, w v_{\text {subt }}^{*}\right)$ obtenidos se calculan una serie de indicadores económicos (valor de la producción, margen neto, empleo agrario, coste para la Administración) que permitan evaluar los diferentes escenarios de gestión propuestos. Los indicadores económicos se han calculado de la siguiente forma:

- Valor de la producción agraria:

- Margen neto:

- Empleo agrario:

- Coste a asumir por la Administración:

Donde: $P F A(w u)$ es una función que liga la cantidad de agua utilizada en la zona con el valor de la producción agrícola de regadío generado en la misma (euros); $M N(w u)$ es una función que liga la cantidad de agua utilizada en la zona con el margen neto generado (euros); $M O(w u)$ es una función que liga la cantidad de agua utilizada en la zona con la mano de obra agraria utilizada (euros). Estas tres funciones provienen de un modelo no-lineal de optimización que simula las decisiones de asignación de tierra y agua entre cultivos en el regadío de la cuenca del Segura y genera indicadores económicos de uso del agua. Una descripción detallada de este modelo, incluyendo su estructura matemática, sus coeficientes técnicos y económicos y su calibración, puede verse en Martínez-Granados et al. (2011) y Calatrava y Martínez-Granados (2012).

En el caso del escenario de no intervención el modelo se modifica tomando valores nulos para el precio de compra del derecho y la cantidad de derechos adquiridos $\left(p c=0 ; w v_{\text {subt }}=\right.$ $0)$. En el caso del escenario de prohibición de extracciones, además de lo anterior, el uso de aguas subterráneas se restringe a su fracción renovable substituyendo la restricción [4] por: de recursos subterráneos ( $\left.\mathrm{hm}^{3} / a n ̃ o\right)$. donde nonrenov son las extracciones medias no renovables

\section{4- Resultados}

En primer lugar, los resultados para el escenario de "no intervención" (segunda columna de la Tabla 2) muestran cómo, debido a la elevada demanda de agua de riego en la zona, se utilizarían aproximadamente la cuarta parte de los recursos desalinizados disponibles (en concreto, 7,44 de $28 \mathrm{hm}^{3}$ anuales), pese a que su precio es comparativamente superior (ver Tabla 1). Sin embargo, este incremento en el uso de recursos desalinizados no supondría una reducción en las extracciones del acuífero debido al notable grado de escasez de agua de la zona. En esta situación, el valor de la producción agraria es de aproximadamente 202 millones de euros/año, el margen neto de 64,6 millones de euros/año y el empleo agrario generado de 1,263 millones de jornales anuales.

Una opción para eliminar la sobreexplotación de acuíferos es limitar el uso de recursos subterráneos a la fracción renovable de los bombeos. En una situación de no disponibilidad de recursos desalinizados, el impacto económico sería notable, ya que los recursos subterráneos no renovables suponen más de una tercio de la disponibilidad media de agua 
para regadío en la zona. Sin embargo, puesto que en el futuro inmediato los regantes podrán disponer de recursos procedentes de la desalinización, el impacto de prohibir las extracciones no renovables se vería limitado, ya que los regantes podrían sustituir el agua subterránea por agua desalinizada y casi todos los recursos desalinizados disponibles se utilizarían pese a ser más caros que los subterráneos. Como se muestra en la tercera columna de la Tabla 2, en este escenario se reduce el valor de la producción agraria en 12,5 millones de euros/año (un 6,2\%), el margen neto agrario en 6 millones de euros/año (un 9,2\%) y el empleo agrario en 113.900 jornales al año (un 9,0\%). La disponibilidad de agua desalinizada compensa en parte el impacto negativo que la restricción de las extracciones de recursos subterráneos tendría para el sector agrario de la zona.

Tabla 2. Impacto económico de la eliminación de la sobreexplotación en el acuífero "Alto Guadalentín" mediante la restricción de bombeos y la compra pública de derechos

\begin{tabular}{|c|c|c|c|}
\hline & $\begin{array}{l}\text { No intervención } \\
\text { (situación actual) }\end{array}$ & $\begin{array}{l}\text { Prohibición de } \\
\text { extracciones no } \\
\text { renovables }\end{array}$ & $\begin{array}{l}\text { Adquisición de } \\
\text { derechos }(0,38 \\
\left.\text { euros } / \mathrm{m}^{3} / \mathrm{año}\right)\end{array}$ \\
\hline Uso total de agua ( $\left.\mathrm{hm}^{3} / a n ̃ o\right)$ & 81,88 & \multicolumn{2}{|c|}{$\begin{array}{c}74,44 \\
(-9,1 \%) \\
\end{array}$} \\
\hline $\begin{array}{l}\text { Uso de aguas subterráneas } \\
\left.\text { ( } \mathrm{hm}^{3} / a n ̃ o\right)\end{array}$ & 36,32 & \multicolumn{2}{|c|}{$\begin{array}{c}8,72 \\
(-76,0 \%)\end{array}$} \\
\hline $\begin{array}{l}\text { Reducción de extracciones } \\
\left(\mathrm{hm}^{3} / \mathrm{anno}\right)\end{array}$ & 0,00 & \multicolumn{2}{|c|}{27,60} \\
\hline $\begin{array}{l}\text { Uso de agua desalinizada } \\
\text { ( } \mathrm{hm}^{3} \text { /año) }\end{array}$ & 7,44 & \multicolumn{2}{|c|}{$\begin{array}{c}27,60 \\
(+271,0 \%) \\
\end{array}$} \\
\hline $\begin{array}{l}\text { Producción agraria (millones } \\
\text { de euros/año) }\end{array}$ & 201,97 & \multicolumn{2}{|c|}{$\begin{array}{l}189,51 \\
(-6,2 \%) \\
\end{array}$} \\
\hline $\begin{array}{l}\text { Margen neto agrario (millones } \\
\text { de euros/año) }\end{array}$ & 64,59 & $\begin{array}{c}58,62 \\
(-9,2 \%)\end{array}$ & $\begin{array}{c}69,11 \\
(+6,9 \%)\end{array}$ \\
\hline $\begin{array}{l}\text { Empleo agrario }\left(10^{4}\right. \\
\text { jornales/año) }\end{array}$ & 126,26 & \multicolumn{2}{|c|}{$\begin{array}{l}114,87 \\
(-9,0 \%)\end{array}$} \\
\hline $\begin{array}{l}\text { Coste presupuestario público } \\
\text { (millones de euros/año) }\end{array}$ & 0,00 & 0,00 & 10,47 \\
\hline
\end{tabular}

Fuente: Elaboración propia. Cambio porcentual con respecto a la situación actual entre paréntesis.

De manera similar, la adquisición pública de derechos tendría un impacto menor de lo que sería esperado, al existir la posibilidad de sustituir derechos vendidos por recursos desalados (cuarta columna de la Tabla 2). La Figura 2 muestra cómo, a medida que se incrementa el precio de adquisición del derecho de agua subterránea, se reducen las extracciones (que son sustituidas en gran medida por recursos desalinizados) y, paralelamente, disminuye el valor de la producción agraria, mientras que se incrementan tanto el margen neto de los agricultores como el coste presupuestario soportado por la Administración derivado de la compra de los derechos. El empleo agrario evoluciona en paralelo al valor de la producción agraria, por lo que en la Figura 2 solo se muestra esta última para simplificar la figura.

La eliminación de la sobreexplotación en el acuífero Alto Guadalentín mediante la adquisición pública de derechos de agua exigiría alcanzar un precio de compra de 0,38 $€ / \mathrm{m}^{3} / a n ̃ o$, medido como una coste anual equivalente. Como consecuencia, el impacto en términos de producción y empleo agrario sería el mismo que en el escenario anterior. Por el contrario, en lugar de reducir el margen neto, la adquisición de derechos supondría un incremento de la rentabilidad privada de los agricultores de 4,5 millones de euros anuales (un 6,9\%), si bien a costa de un gasto público de 10,5 millones de euros anuales. Si se capitaliza dicho coste anual considerando un tipo de interés de entre 0,03 y 0,05 se obtiene un coste total de la OPAD de entre 210 y 350 millones de euros respectivamente. 


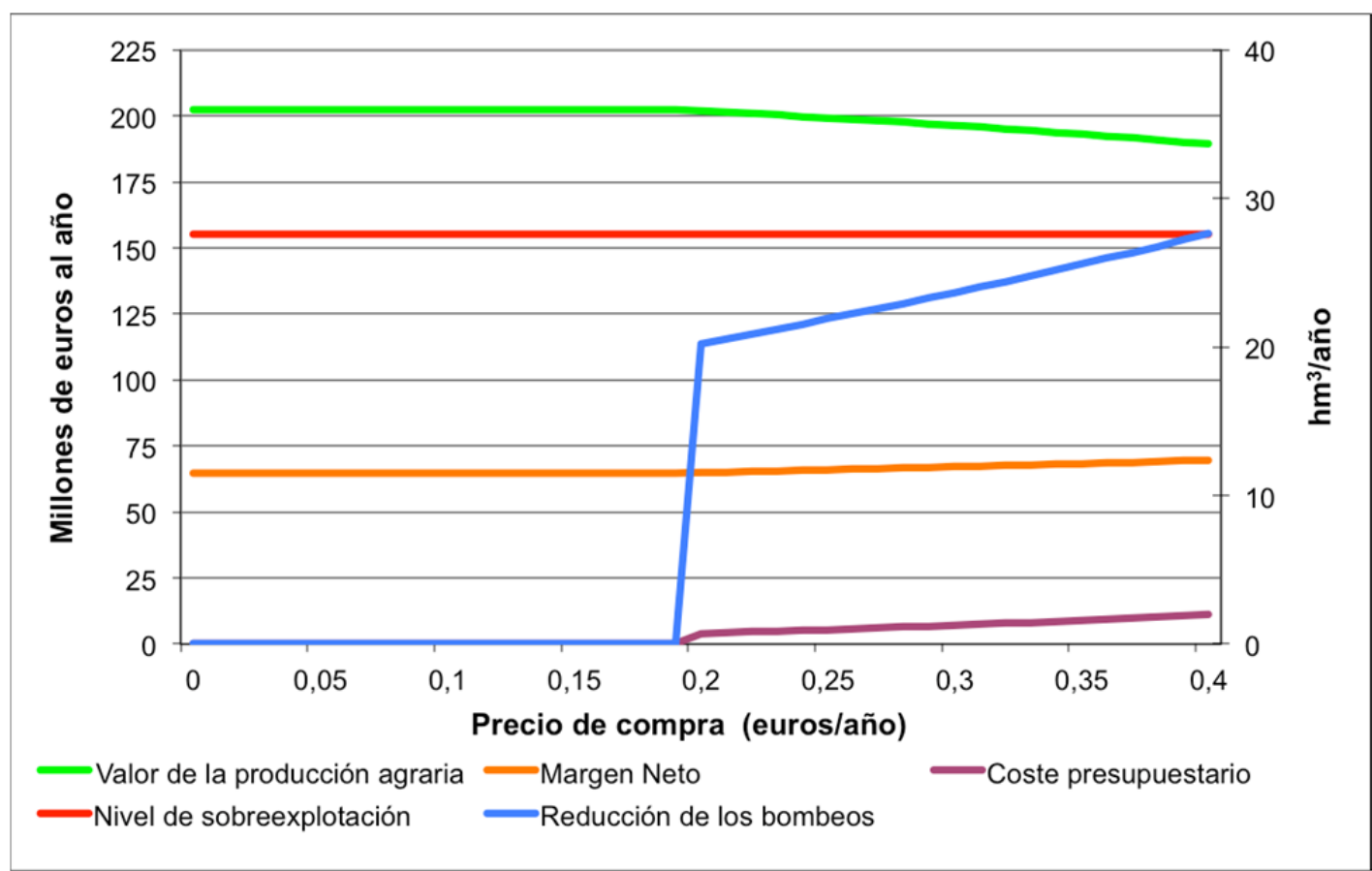

Figura 2. Impacto de la compra de derechos sobre las extracciones de aguas subterráneas, la producción, el margen neto y el coste presupuestario público. Elaboración propia.

\section{5- Conclusiones}

La sobreexplotación de acuíferos es uno de los principales problemas ambientales y de gestión del agua en el sureste Español, siendo especialmente grave en el acuífero Alto Guadalentín en la cuenca del Segura. Frente a medidas de mando y control basadas en la restricción de las extracciones, una de las alternativas utilizadas en España para hacer frente a este problema es la adquisición pública de derechos de aguas subterráneas. Este trabajo analiza el impacto económico de eliminar la sobreexplotación en el acuífero Alto Guadalentín mediante la adquisición de derechos de aguas subterráneas, en términos de rentabilidad de la actividad agraria, impacto social y coste presupuestario, y teniendo en cuenta la futura disponibilidad de recursos procedentes de la desalinización de agua de mar.

En primer lugar, nuestros resultados muestran como la eliminación de las extracciones no renovables tiene importantes efectos económicos sobre el sector agrario, aunque la disponibilidad de recursos desalinizados los reduce significativamente, si bien no los elimina totalmente, ya que los agricultores pueden sustituir recursos subterráneos por desalinizados.

Una primera opción analizada para eliminar la sobreexplotación de acuíferos es restringir las extracciones al nivel de recarga del acuífero mediante la prohibición del uso de los recursos subterráneos que corresponden a bombeos no renovables. En una situación como la actual, en la que el acuífero es una importante fuente de suministro y no existen todavía recursos alternativos, esta alternativa tendría importantes consecuencias negativas, tanto económicas como sociales, sobre el sector agrario al verse reducido el uso de agua. Sin embargo, si consideramos la inminente disponibilidad de recursos procedentes de la desalinización en la zona, el impacto negativo de la prohibición se vería reducido, ya que los agricultores podrían sustituir parcialmente los recursos subterráneos por desalinizados.

Por su parte, la opción de adquisición de derechos de agua de riego por parte de la Administración tiene cierto atractivo, ya que resuelve el problema de la sobreexplotación de manera definitiva y no supone merma alguna para la renta de los agricultores que son 
compensados. Sin embargo, se trata de una alternativa de elevado coste presupuestario y con un notable impacto social en términos de reducción del valor de la producción y el empleo agrarios (similar al de la restricción de las extracciones). De hecho, la diferencia entre ambas alternativas es que en el caso de la restricción de las extracciones el coste de eliminar la sobreexplotación es asumido por los agricultores, mientas que en el caso de la OPAD lo asumiría la Administración.

Aunque la restricción de las extracciones presenta un menor coste presupuestario, se trata de una medida impopular y hasta cierto punto difícil de materializar políticamente. Debe tenerse en cuenta que el éxito de cualquier intervención pública en materia de recursos hídricos depende en gran medida de la cooperación de los usuarios afectados. En este sentido, la adquisición de derechos de agua puede ser mejor recibida por los agricultores que la prohibición de las extracciones, máxime en una situación en la que existe la alternativa del recurso a la desalinización. Es improbable que pudiese aplicarse una medida restrictiva de este tipo sin establecer algún tipo de compensación para los agricultores, como la experiencia de otras cuencas española ha mostrado (Esteban and Albiac, 2011; Garrido et al., 2013). Pese a su elevado coste presupuestario, la adquisición de derechos tiene el mismo impacto sobre el sector agrario, pero es potencialmente menos conflictiva. Una solución intermedia podría ser la combinación de alternativas (incluyendo cierto grado de restricción de extracciones, compra de derechos y subvención a la desalinización) que permitiese repartir el coste de recuperación del acuífero entre agricultores y Administración.

\section{6- Agradecimientos}

La realización de este trabajo ha sido posible gracias a la financiación proporcionada por el Ministerio de Economía y Competitividad y el Fondo Europeo de Desarrollo Regional mediante el proyecto MERCAGUA (AGL2013-48080-C2-2-R).

\section{7- Referencias}

Albiac, J., Hanemann, M., Calatrava, J., Uche, J. \& Tapia, J. (2006). The rise and fall of the Ebro water transfer. Natural Resources Journal, 46(3), 727-757.

Albiac, J., Tapia, J., Meyer, A., Hanemann, M., Mema, M., Calatrava, J., Uche, J. \& Calvo, E. (2008). Los problemas económicos de la planificación hidrológica. Revista de Economía Aplicada, XVI(47), 25-50.

Calatrava, J. \& Gómez-Ramos, A. (2009). "El papel de los mercados de agua como instrumento de asignación de recursos hídricos en el regadío español"; en GómezLimón, J. A., Calatrava, J., Garrido, A., Sáez, F. J. \& Xabadia, À., eds.: La economía del agua de riego en España. Fundación Cajamar, Almería. pp 295-319.

Calatrava, J., Barberá, G.G. \& Castillo, V.M. (2011). Farming practices and policy measures for agricultural soil conservation in semi-arid Mediterranean areas: The case of the Guadalentín basin in southeast Spain. Land Degradation and Development, 22(1), 58-69.

Calatrava, J. \& Martínez-Granados, D. (2012). El valor de uso del agua en el regadío de la cuenca del Segura y en las zonas regables del trasvase Tajo-Segura. Economía Agraria y Recursos Naturales, 12(1), 5-32.

Calatrava, J. \& Martínez-Granados, D. (2016). "Los mercados formales de agua en la cuenca del Segura". En: Gómez-Limón, J. A. y Calatrava, J. (editores) "Los mercados de agua en España: presente y perspectivas". Cajamar Caja Rural, Almería.

Carmona, G., Varela-Ortega, C. \& Bromley, J. (2011). The Use of Participatory ObjectOriented Bayesian Networks and Agro-Economic Models for Groundwater Management in Spain. Water Resources Management, 25(5),1509-1524.

CHS (2008). Esquema provisional de temas importantes de la Demarcación Hidrográfica del Segura. Confederación Hidrográfica del Segura, Murcia. 
Downward, S.R. \& Taylor, R. (2007). An assessment of Spain's Programa AGUA and its implications for sustainable water management in the province of Almería, southeast Spain. Journal of Environmental Management, 82(2), 277-289.

Esteban, E. \& Albiac, J. (2011). Groundwater and ecosystems damages: Questioning the Gisser-Sánchez effect. Ecological Economics, 70(11), 2062-2069.

Garrido, A., Rey, D. \& Calatrava, J. (2013): Water trading in Spain; en De Stefano, L. \& Llamas, M. R., eds.: Water, agriculture and the environment in Spain: Can we square the circle? Botín Foundation, CRC Press, Boca Raton (CA); pp. 205-216.

Hellegers, P. \& van lerland, E. (2003). Policy instruments for groundwater management in the Netherlands. Environmental and Resource Economics, 26(1), 163-172.

Iglesias, E. (2002). La gestión de las aguas subterráneas en el acuífero Mancha Occidental. Economía Agraria y Recursos Naturales, 2(1), 69-88.

Martínez-Granados, D., Maestre-Valero, J.F., Calatrava, J. \& Martínez-Álvarez, V. (2011). The economic impact of water evaporation losses from water reservoirs in the Segura basin, SE Spain. Water Resources Management, 25(13), 3153-3175.

Rey, D., Garrido, A. \& Calatrava, J. (2014). "Water markets in Spain: Meeting twenty-first century challenges with twentieth century regulations"; en Easter, K. W. \& Huang, Q., eds.: Water markets for the 21st. century: What have we learned? Global Issues in Water Policy Series Volume 11. Springer, Dordrecht, The Netherlands; pp. 127-147. 